\title{
Maternal Outcome of Hypothyroidism in Pregnancy- a South Indian Perspect
}

\author{
Nirmala $C^{1}$, Jayakumari $C^{2}$, Rajasekharan $C^{3, *}$, Nandini VR $^{4}$ \\ ${ }^{1}$ Professor and Head, Department of Obstetrics and Gynecology, Sree Avittom Thirunal Hospital, Medical College, \\ Thiruvananthapuram \\ ${ }^{2}$ Associate Professor of Medicine, Department of Medicine, Medical College Hospital, Thiruvananthapuram \\ ${ }^{3}$ Professor of Medicine, Department of Medicine, Medical College Hospital Thiruvananthapuram \\ ${ }^{4}$ Associate Professor, Department of Obstetrics and Gynecology, Sree Avittam Thirunal Hospital, Medical College \\ *Corresponding author: drcrajasekharan@yahoo.com
}

Received December 11, 2013; Revised March 08, 2014; Accepted March 10, 2014

\begin{abstract}
Background: Hypothyroidism in pregnancy is increasing and is a major cause for concern because of its association with adverse maternal outcome if untreated. The literature related to the burden of adverse pregnancy outcomes associated with hypothyroid pregnant women on thyroxine replacement therapy is limited. Hence, this study was designed. Objective: The primary objective was to study the maternal outcome of hypothyroidism in pregnancy. Methodology: This study was conducted in the Department of Obstetrics \& Gynecology in Govt. Medical College Trivandrum. This was a mixed method study that included retrospective patient data collection and a prospective matched case control study. Seventy-eight pregnant women were recruited as cases based on diagnosis of hypothyroidism prior to pregnancy from the medical department records. They were compared with 78 euthyroid pregnant women, approximately age matched. History and pregnancy outcome were assessed. Data was analyzed using SPSS statistical package. Results: Hypothyroidism was associated with 3.6 times higher risk of developing threatened abortion and 3.8 times higher risk of developing gestational hypertension $(\mathrm{P}=0.001)$. Rate of caesarian section (51.3\%) and postpartum hemorrhage (12.8\%) were higher among cases. Conclusions: Based on our study we conclude that hypothyroid mothers, in spite of thyroid replacement therapy, are at significantly higher risk of obstetric complications. A multidisciplinary team approach, where the services of an endocrinologist and an obstetrician experienced in high risk pregnancy care are used, and a tertiary level institution with good facilities for ante partum and intrapartum care, is essential to improve the outcomes.
\end{abstract}

Keywords: endocrine, hypothyroidism, pregnancy outcome, pregnancy complication, maternal thyroid dysfunction

Cite This Article: Nirmala C, Jayakumari C, Rajasekharan C, and Nandini VR, "Maternal Outcome of Hypothyroidism in Pregnancy- a South Indian Perspect.” American Journal of Clinical Medicine Research, vol. 2, no. 2 (2014): 47-50. doi: 10.12691/ajcmr-2-2-2.

\section{Introduction}

A normal pregnancy results in a number of important physiological and hormonal changes that alter thyroid function. Alterations in the thyroid functions are due to the influence of two hormones- human chorionic gonadotrophin (HCG) and estrogen [1]. Estrogen increases the thyroid binding protein in the serum, which increases the total thyroid hormone levels in the blood, since $>99 \%$ of the thyroid hormone in the blood are bound to these proteins [2]. Serum Thyroid Binding globulin (TBG) increases a few weeks after conception and reaches a plateau during mid gestation. The mechanism for increase in TBG involves both an increase in hepatic synthesis of TBG and an oestrogen induced increase in the half life of TBG (from 15 min to 3 days) [3]. The levels of maternal thyroid concentrations, both total thyroxine (TT4), and total triiodothyronine (TT3) increase from early pregnancy [4]. The high circulating of levels of HCG in the first trimester may result in a slightly low TSH [2]. For the first 10-12 weeks of pregnancy the baby is completely dependent on the mother for the production of thyroid hormones. The first 12 weeks is the crucial period in fetal and placental development. In human fetal brain thyroid hormone receptors, TR $\alpha 1$ and TR $\beta 1$ isoforms and receptor binding are present by 8-10 weeks. TR $\alpha 1$ isoforms increase 8-10 fold by $16-18$ weeks [5]. Studies suggest that, children of even marginally iodine deficient mothers show psychomotor and cognitive impairment Such data indicates sensitivity of the developing CNS to maternal thyroid metabolism in utero [6]. By the end of the first trimester, the baby's thyroid gland begins to produce thyroid hormone its own.

The baby remains dependent on the mother for ingestion of adequate amount of iodine which is essential to make thyroid hormone [7]. World Health Organisation (WHO) recommends iodine intake of 150 micrograms/days for non-pregnant women, for pregnant and lactating women it is $200-250$ micro-grams/day to maintain adequate thyroid hormone production [8]. 
Over the past several years it has been shown that thyroid function abnormality in the mother affects the outcome of the fetus and the mother during and also after delivery [9].

Untreated hypothyroidism in pregnancy has been associated with miscarriage, pre-eclampsia, post partum hemorrhage [10]. Severe iodine deficiency during pregnancy causes maternal and foetal hypothyroidism which can result in irreversible brain damage. [11] Hypothyroidism in pregnancy can be defined as subclinical (normal Free Tetra iodothyronine $\left(\mathrm{FT}_{4}\right)$ and high TSH) or overt hypothyroidism (low $\mathrm{FT}_{4}$ and high TSH).

There can be a varied presentation of hypothyroidism in women during pregnancy. Many of the symptoms and signs of hypothyroidism can overlap with pregnancy, like lethargy weakness, muscle cramps, constipation, hair loss etc, Thus it is difficult to relay on symptoms to make a diagnosis of hypothyroidism in pregnancy [2].

Once hypothyroidism has been detected, the women should be treated with levothyroxine to normalize her TSH and free T4 values. The levothyroxine requirements frequently increase during pregnancy [12]. Ideally, hypothyroid women should have their thyroxine dose optimized and become euthyroid before they conceive [13]. There is an increase in the incidence of hypothyroidism in younger population particularly in the reproductive age women. These women have problems with conception. Many of them are diagnosed prior to pregnancy and are on replacement therapy. There is little information related to the pregnancy outcome of hypothyroid pregnant women on replacement therapy. Hence this study was undertaken to assess the maternal problems associated with hypothyroidism in pregnancy who were diagnosed prior to pregnancy, and on Lthyroxine replacement.

\section{Aims and Objectives}

To study the maternal outcome of hypothyroid pregnant women on L- thyroxine replacement therapy.

\section{Methodology}

This study was conducted in the Department of Obstetrics \& Gynecology in Govt. Medical College, Trivandrum, Kerala, in collaboration with the Department of Medicine. The study period was one year from Jan 2011- Dec 2011. This was a mixed method study that included retrospective patient data collection and a prospective matched case control design. Cases were identified from the medical records in the thyroid clinic attached to the Department of Medicine. Universal sampling technique was used.

All consecutive pregnant women with singleton pregnancy diagnosed as hypothyroidism prior to conception and on L-thyroxine replacement therapy who consented to participate in the study were included as cases. Their pregnancy was registered in the antenatal clinic of the Department of Obstetrics \& Gynecology and followed up with the routine antenatal care. For each case, an age matched pregnant women with no evidence of hypothyroidism was chosen as control. Pregnant women with any medical illness or any pregnancy related complications like gestational diabetes, hypertension, preeclampsia, bad obstetric history etc were excluded from the study. All women were registered in the first trimester. A total of 78 cases and 78 controls were recruited for the study and followed up till delivery. Ethical clearance was obtained from Institutional ethical committee.

After getting an informed consent, a detailed history regarding age, socioeconomic status, menstrual and obstetric history were taken using a structured questionnaire. Details related to duration of hypothyroidism and treatment history were also collected. A thorough general physical examination and obstetric examination were carried out. Routine antenatal examination and investigations including the thyroid function were assessed. They were all screened for TORCH infection. Ultrasound examinations were done at 18-20 weeks for gestational age, and to rule out major structural anomalies.

Maternal outcomes studied were threatened abortion, preterm delivery, gestational hypertension, gestational diabetes, mode of delivery and postpartum haemorrhage.

The data were tabulated on a spread sheet and analyzed with SPSS statistical package. We calculated mean and standard deviation for continuous variables and proportion for other parameters. Means for the group were compared by t-test and proportion using Chi-square test. Odds Ratio and $P$ value were used to find out the significant difference. $\mathrm{P}$ value of $<0.05$ was considered as significant.

Table 1. Demographic characteristics

\begin{tabular}{|c|c|c|c|c|c|}
\hline & & \multicolumn{2}{|c|}{ Case } & \multicolumn{2}{|c|}{ Control } \\
\hline \multicolumn{2}{|c|}{ Study variables } & $\mathrm{N}$ & $\%$ & $\mathrm{~N}$ & $\%$ \\
\hline \multirow[t]{2}{*}{ Age } & $\begin{array}{c}<30 \\
\text { years }\end{array}$ & 56 & 71.8 & 62 & 79 \\
\hline & $\begin{array}{c}>30 \\
\text { years }\end{array}$ & 22 & 28.2 & 16 & 20.5 \\
\hline \multirow[t]{3}{*}{ Religion } & Hindu & 44 & 56.4 & 50 & 64.1 \\
\hline & Muslim & 18 & 23.1 & 13 & 16.7 \\
\hline & Christian & 16 & 20.5 & 15 & 19.2 \\
\hline \multirow[t]{4}{*}{ Parity } & Primi & 45 & 57.7 & 38 & 48.7 \\
\hline & P1 & & & & \\
\hline & $\mathrm{P} 2$ & & & & \\
\hline & P3 & 7 & 5.1 & 4 & 5.1 \\
\hline Socio & High & 18 & 23.1 & 13 & 16.7 \\
\hline economic & Middle & 45 & 57.7 & 41 & 52.6 \\
\hline status & Low & 15 & 19.2 & 24 & 30.8 \\
\hline
\end{tabular}

\section{Results}

The demographic and clinical characteristics of hypothyroid and euthyroid groups were compared in Table 1. There were 78 subjects with hypothyroidism in pregnancy and 78 pregnant controls without hypothyroidism. Their mean age was 26.29 years, median age 25.5 years. (range 18-33 years).

There was no difference between the two groups with regard to parity, socio-economic status and religion or booking status. Maternal outcomes in cases and controls are compared in Table 2. 23.1\% of cases developed threatened abortion as compared to $7.7 \%$ of controls. (Odds ratio 3.6; 95\% CI 1.34-9.64). 35.9\% of cases and $12.8 \%$ of controls developed gestational hypertension, suggesting that hypothyroidism in pregnancy has 3.8 
times higher risk of developing gestational hypertension as compared to controls (Odds ratio 3.8; 95\% CI 1.7 - 8.6; $P$ 0.001). Preterm labor was seen in $25.6 \%$ of cases and $20.5 \%$ of controls and the difference were not statistically significant. Postpartum haemorrhage was seen in $12.8 \%$ of cases and $3.8 \%$ of controls, but did not show a statistically significant association. Caesarean section rate was higher in cases. $51.3 \%$ of cases and $28.2 \%$ of controls had caesarean section. Gestational hypertension and Caesarean section are found to be significant during the multivariate analysis.

Table 2. Distribution of Matemal Outcomes

\begin{tabular}{|c|c|c|c|c|c|c|c|}
\hline & \multicolumn{2}{|c|}{ Case } & \multicolumn{2}{c|}{ Control } & & & \\
\hline Variables & $\mathrm{N}$ & $\%$ & $\mathrm{~N}$ & $\%$ & $\mathrm{p}$ & $\mathrm{OR}$ & $95 \% \mathrm{CI}$ \\
\hline Threatened & & & & & & & \\
\hline Abortion & 18 & 23.1 & 6 & 7.7 & 0.007 & 3.6 & $1.3-9.6$ \\
\hline Gestational & & & & & & & \\
\hline Hypertension & 28 & 35.9 & 10 & 12.8 & 0.001 & 3.8 & $1.7-8.6$ \\
\hline Postpartum & & & & & & & \\
\hline hemorrhage & 10 & 12.8 & 3 & 3.8 & 0.039 & 3.7 & $1.0-13.9$ \\
\hline Caesarean & & & & & & & \\
\hline section & 40 & 51.3 & 22 & 28.2 & 0.003 & 2.7 & $1.4-5.2$ \\
\hline
\end{tabular}

Table 3. binary logistic regression analysis

\begin{tabular}{|c|c|c|c|c|c|c|}
\hline & & & & & \multicolumn{2}{c|}{$95 \%$ C.I. for OR } \\
\hline Variables & B & S.E. & p & OR & Lower & Upper \\
\hline Threatened & 1.033 & 0.537 & 0.054 & 2.809 & 0.981 & 8.04 \\
\hline Abortion & & & & & & \\
\hline Gestational & 1.029 & 0.437 & 0.019 & 2.799 & 1.188 & 6.596 \\
\hline Hypertension & & & & & & \\
\hline Postpartum & 1.331 & 0.723 & 0.066 & 3.785 & 0.917 & 15.619 \\
\hline hemorrhage & & & & & & \\
\hline Caesarean & 0.834 & 0.361 & 0.021 & 2.303 & 1.134 & 4.676 \\
\hline section & & & & & & \\
\hline Constant & 3.428 & 0.945 & 0 & 0.032 & & \\
\hline
\end{tabular}

\section{Discussion}

This study provides us an opportunity to study the reproductive outcome of hypothyroidism in pregnancy, diagnosed prior to pregnancy and on treatment with Lthyroxine. The key observation from this study was the significantly increased adverse maternal outcomes in hypothyroid cases as compared to those without hypothyroidism. Both groups were comparable in terms of age, parity and socioeconomic status. There was 3.8 times higher risk of developing gestational hypertension in the case (35.9\%) when compared to controls (12.8\%) A previous study by Negro et al reveals that, untreated thyroid dysfunction in pregnancy results in an increased adverse events particularly gestational hypertension and preterm birth [14]. Postpartum hemorrhage complicated $12.8 \%$ of the cases and $3.8 \%$ of the controls. The postpartum haemorrhage in hypothyroidism is produced both through the uterine hypotony and coagulation problems with plaque adhesiveness problem. Various studies from literature indicate a percentage between 7 and $19 \%$ [15].

The significantly higher incidence of gestational hypertension and postpartum hemorrhage among hypothyroid pregnant women has important public health implications. Postpartum hemorrhage and gestational hypertension are the major contributors of maternal mortality in Kerala. Thus hypothyroidism indirectly contributes to maternal mortality and morbidity.
Those with hypothyroidism had 3.6 times the risk of developing threatened abortion. Several studies reveals that impaired thyroid function may predispose to miscarriage and foetal death. $[10,16,17]$ Rate of Caesarean section was higher among hypothyroid (51.3\% of cases and $28.2 \%$ of controls). The rate of preterm delivery is also high in this group (25.6\%). Rate of low birth weight babies were higher among cases (28.2\%) this shows that hypothyroidism is a risk factor for early delivery of the baby. Previous studies also show that hypothyroidism is associated with premature birth and low birth weight babies. [18] Preterm birth and low birth weight are major cause neonatal mortality and morbidity.

\section{Conclusion}

Based on our study we conclude that hypothyroid mothers are at significantly higher risk of obstetric complications in spite being diagnosed prior to pregnancy and on thyroid replacement. A multidisciplinary team approach where the services of an endocrinologist, obstetrician experienced in high risk pregnancy care and a tertiary level institution with good facilities for ante partum and intrapartum care are essential to improve the outcomes.

\section{References}

[1] Gaberscek S, Zaletel K. Thyroid physiology and auto immunity in pregnancy and after delivery. Expert Rev Clin Immunol. 2011; Vol. 7 (5) 697-706.

[2] Dharmalingam M, KalraP, Mittal R, Jayakumar RV (eds). Thyroid diseases during prgnency, Indian Thyroid Society, Clinical Manual of Thyroid Disorders,1st edition, New Delhi, ReedElsevier India 2012; 222-23

[3] Corinne. R.F, Samuel DA, Jack. H.L and Ann. M.G, Thyroid function during pregnancy, Clinical Chemistry 1999; 45: 12 22502258.

[4] Lazarus JH, Mestman JH. In Thyroid disorders during pregnancy and post partum. Braverman THE THYROID A Fundamental and Clinical Text, 10th edition Vol. 2 (sec. 4-8) pg. 815.

[5] Kota SK, Gayathri K, Jammula S, Mehar LK, Kota SK, Krishna SV. Review Article Foetal endocrinology, In. Journal of Endocrinology and Metabolism 2013: vol. 17 issues 4 Pg 568-579.

[6] S. Chan, Kilby Thyroid Hormone and Central Nervous System development. J Endocrinol. Review (2000) 165 1-8.

[7] Thyroid disease and Pregnanacy. The American Thyroid Association Available from http:/www.thyroid.org/patient brochures/thyroid Dis. pregnancy broch.pdf (accessed on Nov. 2011).

[8] Iodine requirements in pregnancy and infancy. A WHO Technical Consultation report on new guidelines on iodine requirements. IDD Newsletter. 2007; Vol. 23 (1): 1-20.

[9] Berbel P, Mestre JL, Santamaría A, Palazón I, Franco A, Graells $\mathrm{M}$, etal. Delayed neurobehavioral development in children born to pregnant women with mild hypothyroxinemia during the first month of gestation: the importance of early iodine supplementation. Thyroid.2009May; 19 (5): 511-9.

[10] LaFranchi SH, Haddow JE, Hollowell JG is thyroid inadequacy during gestation a risk factor for adverse pregnancy and developmental outcomes? Thyroid. 2005 Jan; 15 (1): 60-71.

[11] Halperson. In. The neuromotor deficit in endemic cretinism and its implication for the pathogenisis of the disorder. Stanbung JB (ed). The damaged brain of iodine deficiency New York Cognizant Communication 1994 15-24.

[12] Yassa. L, Marqusee. E, Fawcett. R, Alexander. EK. Thyroid hormone early adjustment in pregnancy (the therapy) trial. J Clin Endocrinol Metab. 2010 Jul; 95 (7): 3234-41.

[13] Bijay. V, Peter L. Negro. R. Treatment and survey of hypothyroidism during pregnancy: result of a European survey, European journal of endocrinology 166 (149.54). 
[14] Negro R, Mestmen and JH Thyroid disease in pregnancy, Best practice and Research: Clin Endocrinology and metabolism 2011; 25 (6): 927-943.

[15] Tudosa R, Vartej P, Horhoianu I, Ghica C, Mateescu S, Dumitrache I. Maternal and fetal complications of the hypothyroidism- related pregnancy. J Clinical Medicine. Maedica (Buchar). 2010 Apr; 5 (2): 116-23.

[16] Ashoor G, Maiz N, Rotas M, Jawdat F, Nicolaides KH. Maternal thyroid function at 11 to 13 weeks of gestation and subsequent fetal death. Thyroid. 2010 Sep; 20 (9): 989-93.
[17] Nambiar V, Jagtap VS, Sarathi V, Lila AR, Kamalanathan S, Bandgar TR, Menon PS, Shah NS. Prevalence and impact of thyroid disorders on maternal outcome in asian-Indian pregnant women. J Thyroid Res. 2011; 2011: 429097.

[18] Diana. L, Fitzpatrick Michelle A. Diagnosis and Management of Thyroid disease in Pregnancy. Obstetrics and Gynecology, Clinics of North America. June 2010 Vol. 37 No 2 Pg 172-181. 